\section{Antitumor T cells}

The rich repertoire of $\mathrm{T}$ cell receptors is awaiting large-scale profiling.

Harnessing an organism's immune system to fight a disease such as cancer is a powerful approach. In 2018 James Allison and Tasuku Honjo received the Nobel Prize in Physiology or Medicine for their lifetime work on two key immune checkpoint proteins displayed on the surface of T cells. These cell-surface receptors bind certain ligands displayed on other cells such as antigen-presenting cells (APCs) and recognize them as self, which leads to the attenuation of $\mathrm{T}$ cell activity and puts a brake on the immune response. The researchers independently showed during decades of work that preventing these receptors on $\mathrm{T}$ cells from binding their ligands on APCs or tumor cells lifts the block and triggers an attack on the tumor cells.

For full activation of a $\mathrm{T}$ cell, this brake on negative immune modulation is necessary, but not sufficient. Another surface receptor, the T cell receptor (TCR), also needs to recognize and bind a ligand specifically derived from tumor cells and displayed on APCs. The full repertoire of antigens that activate antitumor T cells is only beginning to be discovered as new experimental approaches for profiling these ligands are emerging.

Computational approaches such as TraCeR (Nat. Methods 13, 329-332; 2016) were used to profile the diversity of fulllength TCRs in T cells from single-cell RNA-seq data and were able to pair the reconstructed TCRs, including their antigenbinding sites, with the transcriptional profiles of the cells. This approach allowed the identification of clones specific for the same antigen. Experimental methods use baits to get a handle on TCR ligands. Christopher Garcia and colleagues, for example, used a library of peptide-human leukocyte antigens (pHLAs) displayed on yeast to screen orphan TCRs of tumorinfiltrating lymphocytes. The cognate pHLA bound TCRs with high specificity and allowed the identification of the antigen bound by the tumor-infiltrating T cells (Cell 172, 549-563; 2018).

To fully understand $\mathrm{T}$ cell activation and prevent unwanted negative modulation, scientists will need new wet and dry approaches to profile the receptors on a $T$ cell and the ligands that engage them. The more cell-type-specific ligands we find that activate immune cells, the more likely it will be that receptors on

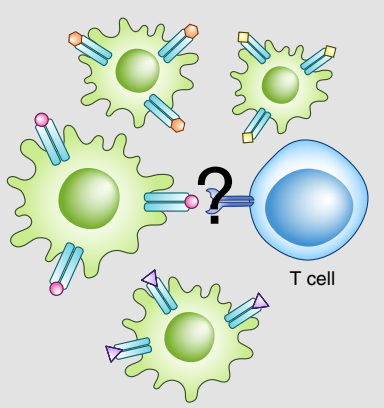

Getting at T cell receptor diversity. Credit: Marina Corral Spence/Springer Nature

T cells could be engineered to target specific cells. In a recent approach, directed by Yvonne Chan at UCLA, TCRs were engineered to respond to specific soluble ligands, instead of only surface-bound ones, which will broaden the repertoire of antigens a $\mathrm{T}$ cell is activated by

(Nat. Chem. Biol. 14, 317-325; 2018).

The ultimate goal is to trigger cell killing only in response to specific ligands, while leaving the rest of the cells unscathed.

Nicole Rusk

https://doi.org/10.1038/s41592-018-0271-0

\section{Microbial interactions}

Synthetic approaches help to dissect the complexity of microbial interactions.

Microorganisms are present in nearly every niche on Earth, from the human gut to the deep ocean, but it is estimated that only $1 \%$ of environmental bacteria are cultivatable. The inability to culture the majority of microbial species has motivated the use of culture-independent methods for studies of microbial diversity, composition, and distribution in different environments. Such methods range from sequencing to imaging techniques such as fluorescence in situ hybridization to mass spectrometry, and have offered metabolic, taxonomic, and spatial profiles of microbial communities.

However, mechanistic understanding of how microbes interact with each other and with their environment has lagged behind. Deconvolution of the sheer complexity of microbial interactions in natural environments has been a major challenge.

Synthetic microbial communities offer reduced complexity and are amenable to mathematical modeling, which makes them a promising resource for studying interactions and their contribution to community structure and function. To this end, advances

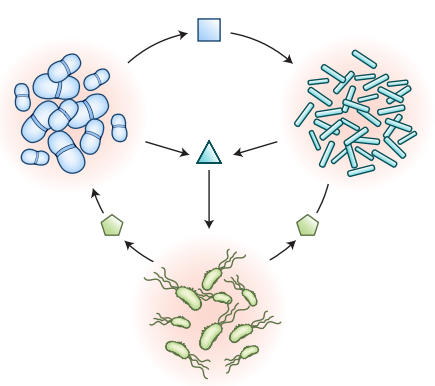

Dynamic interspecies interactions in microbial communities. Credit: Marina Corral Spence/ Springer Nature

in synthetic biology have made it possible to engineer microbes with genetically defined properties. These engineered bacteria have been combined with artificial environments for the study of microbial interactions in response to environmental cues.

In most cases, synthetic approaches involve the coculture of combinations of well-characterized bacterial strains. Although the approach seems straightforward in principle, interactions may be much more complex than simple cooperative associations. Therefore, mathematical modeling is often used to describe and predict these interactions.
For example, a constant yield expectation analytical model is used to deconvolute metabolic interactions in coculture by leveraging the metabolic profiles of monocultures and cocultured strain pairs (Cell Syst. 7, 245-257; 2018).

Experimentally, microchamber-based methods have been developed to mimic the natural environment in which nutrient exchange, water flow, and exposure to oxygen can be crucial for accurate profiling of the microbial community. For example, a microfluidic assay enables assessment of dynamic root-microbe interactions (Proc. Natl Acad. Sci. USA 114, 4549-4554; 2017), and an in situ chemotaxis assay (ISCA) accesses marine microbial behaviors at spatially relevant scales (Nat. Microbiol. 2, 1344-1349; 2017).

Despite the remarkable advances in the study of microbial communities, we are still far from understanding the full picture. Moving beyond purely observational approaches, we look forward to experimental and computational methods that facilitate the interpretation of microbes' interactions with each other and with their communities.

\section{Lei Tang}

https://doi.org/10.1038/s41592-018-0272-z 